\title{
A Technique to Remove Second Order Singularity Application in the Boundary Element Method for Elastoplasticity Plane Stress Analysis
}

\author{
S.A. Bakar and A.L. Saleh \\ Faculty of Civil Engineering, Universiti Teknologi Malaysia, Malaysia. \\ suhaimibakar@utm.my \\ alatif@utm.my
}

\begin{abstract}
This paper presents a technique to establish the strain incremental formulation in the boundary element method applied to elastoplasticity problem. In this technique, the application of second order singularity problem is avoided, and only first order singularity problem is sufficient. The proposed technique is applied to analyse a timber beam structure at the plastic stage. The solution is compared with existing strain formulation method proposed by established publication. The result gives an improved solution compared with the existing method. The proposed technique is a simplified formulation where there is no second order singularity involved in the formulation.
\end{abstract}

\section{Introduction}

A major problem in the boundary element method applied to elastoplasticity problem is a singularity of strain incremental formulation. This singularity comes from Kernel stress derivative and it is a second order singularity.

Telles and Brebbia [1, 2] presents a method to solve the second order singularity problem by expanding the singular integral and reduce to non-singular integral. This method is supported by Kane [3]. Telles and Brebbia [4] also proposed indirect method to solve hypersingular integral. By using transformation method and combine with Guiggiani and Gigante work [5], Leitao, Aliabadi and Rooke [6] extended Telles and Brebbia works to solve the second order singularity problem.

The existing methods to solve the second order singularity problem involve complicated derivation, formulation and calculation. This paper presents a simple technique to avoid the application of second order degree singularity for the elastoplasticity plane stress analysis. 


\section{Displacement increment and strain increment formulations in the boundary element method}

From Swedlow and Cruse [7], the displacement increment formulation is derived from Betti's theorem and virtual work method, and given in equation (1). This equation states that the displacement increment at internal point in the structure is a function of displacement increment and traction increment at the structural boundary, and plastic strain increment at plastic region.

$$
\delta u_{l}^{i}=\int_{\Gamma} u_{l k}^{*} \delta p_{k} d \Gamma-\int_{\Gamma} p_{l k}^{*} \delta u_{k} d \Gamma+\int_{V} \sigma_{i j(l)}^{*} \delta \varepsilon_{i j}^{p} d V
$$

where:

$\delta \mathrm{u}_{1}{ }^{\mathrm{i}}=$ displacement increment at a point ' $\mathrm{i}$ ' in the direction $\mathrm{x}_{\mathrm{l}}$,

$\Gamma$ and $\mathrm{V}$ are the domain of structural boundary and internal domain of structure respectively,

$\delta \mathrm{p}_{\mathrm{k}}$ and $\delta \mathrm{u}_{\mathrm{k}}$ are traction and displacement increments in the direction $\mathrm{x}_{\mathrm{k}}$ respectively, $\mathrm{p}_{\mathrm{lk}}{ }^{*}$ and $\mathrm{u}_{\mathrm{lk}} *$ are virtual traction increment and virtual displacement increment in the direction $\mathrm{x}_{\mathrm{k}}$ respectively,

$\sigma_{\mathrm{ij}(1)} *=$ Kernel stress due to a unit load applied in direction $\mathrm{x}_{\mathrm{l}}$,

$\delta \varepsilon_{\mathrm{ij}}^{\mathrm{p}}=$ Plastic strain increment.

The last integral in equation (1) is a weakly singular integral with first order degree of singularity. The first and second integrals at the right-hand side of equation (1) is also singular if the source point, i, is located at the boundary. This problem was solved by Swedlow and Cruse [7] by considering a small circle placement at source point at the boundary and considering the limit theory, hence equation (1) is reduce to the established boundary element formulation, as follow:

$C_{l k} \delta u_{k}^{i}+\int_{\Gamma} p_{l k}^{*} \delta u_{k} d \Gamma=\int_{\Gamma} u_{l k}^{*} \delta p_{k} d \Gamma+\int_{V} \sigma_{i j(l)}^{*} \delta \varepsilon_{i j}^{p} d V$

where $C_{l k}$ is a constant value which depend on the condition of structural boundary.

By differentiating equation (1), the displacement increment derivative can be expressed as follow:

$$
\left.\frac{\partial\left(\delta u_{l}^{i}\right)}{\partial x_{m}}=\int_{\Gamma}\left[\begin{array}{ll}
\frac{\partial u_{l 1}^{*}}{\partial x_{m}} & \frac{\partial u_{l 2}^{*}}{\partial x_{m}}
\end{array}\right]\left\{\begin{array}{l}
\delta p_{1} \\
\delta p_{2}
\end{array}\right\} d \Gamma-\int_{\Gamma}^{[}\left[\begin{array}{ll}
\frac{\partial p_{l 1}^{*}}{\partial x_{m}} & \frac{\partial p_{l 2}^{*}}{\partial x_{m}}
\end{array}\right]\left\{\begin{array}{l}
\delta u_{1} \\
\delta u_{2}
\end{array}\right\} d \Gamma+\int_{V}^{[} \frac{\partial \sigma_{11(l)}^{*}}{\partial x_{m}} \frac{\partial \sigma_{22(l)}^{*}}{\partial x_{m}} \frac{\partial \sigma_{12(l)}^{*}}{\partial x_{m}}\right]\left[\begin{array}{l}
\delta \varepsilon_{11}^{p} \\
\delta \varepsilon_{22}^{p} \\
2 \delta_{12}^{p}
\end{array}\right\} d V
$$

The derivative of Kernel stress in the last integral of equation (3) is singular with second order. 
The total strain increment is expressed from the basic strain-displacement relationship as follow:

$\delta \varepsilon_{11}=\frac{\partial(\delta u)_{1}}{\partial x_{1}} ; \delta \varepsilon_{22}=\frac{\partial(\delta u)_{2}}{\partial x_{2}} ; \delta \varepsilon_{12}=\frac{\partial(\delta u)_{1}}{\partial x_{2}}+\frac{\partial(\delta u)_{2}}{\partial x_{1}}$

Thus, the total strain increment can be found by substituting equation (3) into equation (4).

\section{Transformation of Second Order Singular Integral into Non- Singular Integral}

From Telles and Brebbia [1], the second order singularity of the last integral in equation (3) is proved can be removed and reduce to non-singular integral. Equation (5) shows the proposed formulation given by Telles and Brebbia expanded from the last integral in equation (3).

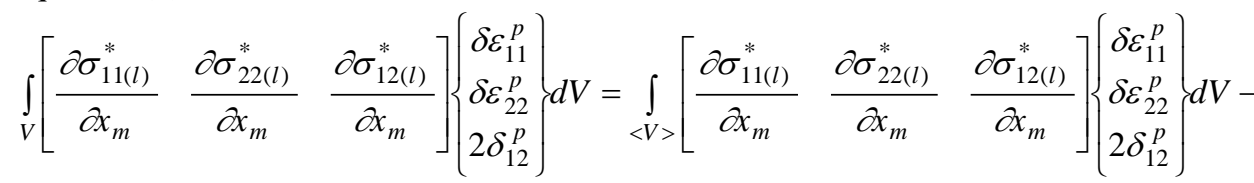

$$
\begin{aligned}
& \int_{\Gamma_{1}}\left[\sigma_{11(l)}^{*} r_{, m} \quad \sigma_{22(l)}^{*} r_{, m} \quad \sigma_{12(l)}^{*} r_{, m}\right] d \Gamma\left\{\begin{array}{c}
\delta \varepsilon_{11}^{p} \\
\delta \varepsilon_{22}^{p} \\
2 \delta \varepsilon_{12}^{p}
\end{array}\right\}
\end{aligned}
$$

where $r$ is a distance between the source point $i$ and observation point, and $r_{, m}=-\partial r / \partial x_{m}$

The first integral at right-hand side of equation (5) can be interpreted in the sense of Cauchy principal value, while the second integral at right-hand side is a boundary integral of a circle of unit radius centered at the source point. Both integrals at right-hand side of equation (5) are non-singular. The reducing of singular integral into non-singular integrals is also supported by Kane [3]. (3), gives:

The displacement derivative is found by substituting equation (5) into equation

$$
\begin{aligned}
& \frac{\partial\left(\delta u_{l}^{i}\right)}{\partial x_{m}}=\int_{\Gamma}\left[\begin{array}{ll}
\frac{\partial u_{l 1}^{*}}{\partial x_{m}} & \frac{\partial u_{l 2}^{*}}{\partial x_{m}}
\end{array}\right]\left\{\begin{array}{l}
\delta p_{1} \\
\delta p_{2}
\end{array}\right\} d \Gamma-\int_{\Gamma}\left[\begin{array}{cc}
\frac{\partial p_{l 1}^{*}}{\partial x_{m}} & \frac{\partial_{l 2}^{*}}{\partial x_{m}}
\end{array}\right]\left\{\begin{array}{l}
\delta u_{1} \\
\delta u_{2}
\end{array}\right\} d \Gamma+ \\
& \int_{<V>}\left[\begin{array}{lll}
\frac{\partial \sigma_{11(l)}^{*}}{\partial x_{m}} & \frac{\partial \sigma_{22(l)}^{*}}{\partial x_{m}} & \frac{\partial \sigma_{12(l)}^{*}}{\partial x_{m}}
\end{array}\right]\left\{\begin{array}{l}
\delta \varepsilon_{11}^{p} \\
\delta \varepsilon_{22}^{p} \\
2 \delta_{12}^{p}
\end{array}\right\} d V-\int_{\Gamma_{1}}\left[\begin{array}{lll}
\sigma_{11(l)}^{*} r_{m} & \sigma_{22(l)}^{*} r_{, m} & \sigma_{12(l)}^{*} r_{, m}
\end{array}\right] d \Gamma\left\{\begin{array}{c}
\delta \varepsilon_{11}^{p} \\
\delta \varepsilon_{22}^{p} \\
2 \delta \varepsilon_{12}^{p}
\end{array}\right\}
\end{aligned}
$$


where $\mathrm{i}$ = source point; $\mathrm{l}$ = direction of point load at source point

The displacement derivative given in equation (3) involved the application of second order singularity problem. One another method, which is proposed in this paper, is a displacement derivative formulation which avoid the application of second order singularity problem.

\section{Proposed strain formulation for the removing of second order singularity problem application}

It is assumed that the internal cell are quadrangular (see Figure 1) where each cell has nine nodes. All functions such as displacement increment are assumed to be quadratic. The displacement increment is related to nodal displacements as follow:

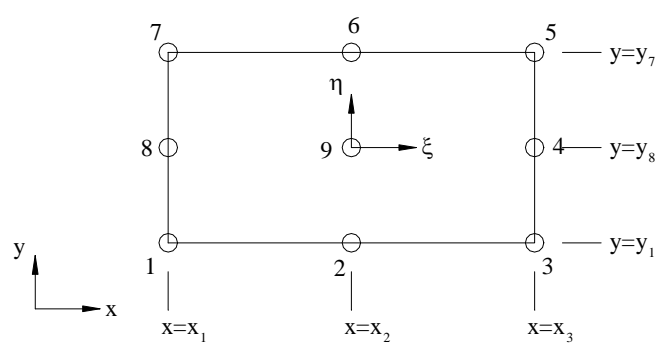

Figure 1. Quadrangular cell at suspected plastic region

$$
\begin{aligned}
& \delta u_{1}=\phi_{1} \delta u_{1}^{1}+\phi_{2} \delta u_{1}^{2}+\phi_{3} \delta u_{1}^{3}+\phi_{4} \delta u_{1}^{4}+\phi_{5} \delta u_{1}^{5}+\phi_{6} \delta u_{1}^{6}+\phi_{7} \delta u_{1}^{7}+\phi_{8} \delta u_{1}^{8}+\phi_{9} \delta u_{1}^{9} \\
& \delta u_{2}=\phi_{1} \delta u_{2}^{1}+\phi_{2} \delta u_{2}^{2}+\phi_{3} \delta u_{2}^{3}+\phi_{4} \delta u_{2}^{4}+\phi_{5} \delta u_{2}^{5}+\phi_{6} \delta u_{2}^{6}+\phi_{7} \delta u_{2}^{7}+\phi_{8} \delta u_{2}^{8}+\phi_{9} \delta u_{2}^{9}
\end{aligned}
$$

where $\phi_{1}, \ldots, \phi_{9}$ are the shape functions, $\delta \mathrm{u}_{1}{ }^{1}, \ldots, \delta \mathrm{u}_{1}{ }^{9}$ are the displacement increment in the direction $\mathrm{x}$ at nodal points, $\delta \mathrm{u}_{2}{ }^{1}, \ldots, \delta \mathrm{u}_{2}{ }^{9}$ are the displacement increment in the direction y at nodal points.

The displacement derivatives with respect to $\mathrm{x}$ and $\mathrm{y}$ axes are found from the chain rule, as follows:

$$
\begin{aligned}
& \frac{\partial\left(\delta u_{1}\right)}{\partial x}=\frac{\partial\left(\delta u_{1}\right)}{\partial \xi} \frac{\partial \xi}{\partial x}+\frac{\partial\left(\delta u_{1}\right)}{\partial \eta} \frac{\partial \eta}{\partial x} \\
& \frac{\partial\left(\delta u_{1}\right)}{\partial y}=\frac{\partial\left(\delta u_{1}\right)}{\partial \xi} \frac{\partial \xi}{\partial y}+\frac{\partial\left(\delta u_{1}\right)}{\partial \eta} \frac{\partial \eta}{\partial y} \\
& \frac{\partial\left(\delta u_{2}\right)}{\partial x}=\frac{\partial\left(\delta u_{2}\right)}{\partial \xi} \frac{\partial \xi}{\partial x}+\frac{\partial\left(\delta u_{2}\right)}{\partial \eta} \frac{\partial \eta}{\partial x} \\
& \frac{\partial\left(\delta u_{2}\right)}{\partial y}=\frac{\partial\left(\delta u_{2}\right)}{\partial \xi} \frac{\partial \xi}{\partial y}+\frac{\partial\left(\delta u_{2}\right)}{\partial \eta} \frac{\partial \eta}{\partial y}
\end{aligned}
$$


Then, the strain formulations are found by substituting equation (8) into equation (4), yields:

$$
\begin{aligned}
& \delta \varepsilon_{11}=\frac{\partial\left(\delta u_{1}\right)}{\partial \xi} \frac{\partial \xi}{\partial x}+\frac{\partial\left(\delta u_{1}\right)}{\partial \eta} \frac{\partial \eta}{\partial x} \\
& \delta \varepsilon_{22}=\frac{\partial\left(\delta u_{2}\right)}{\partial \xi} \frac{\partial \xi}{\partial y}+\frac{\partial\left(\delta u_{2}\right)}{\partial \eta} \frac{\partial \eta}{\partial y} \\
& \delta \varepsilon_{12}=\frac{\partial\left(\delta u_{1}\right)}{\partial \xi} \frac{\partial \xi}{\partial y}+\frac{\partial\left(\delta u_{1}\right)}{\partial \eta} \frac{\partial \eta}{\partial y}+\frac{\partial\left(\delta u_{2}\right)}{\partial \xi} \frac{\partial \xi}{\partial x}+\frac{\partial\left(\delta u_{2}\right)}{\partial \eta} \frac{\partial \eta}{\partial x}
\end{aligned}
$$

By assuming the distance $\mathrm{x}$ and $\mathrm{y}$ are linearly related to respective local distance $\xi$ and $\eta$, the strain functions in equation (9) can be related to nodal displacement increment as follow:

$$
\begin{aligned}
\delta \varepsilon_{11}= & \frac{2}{x_{3}-x_{1}}\left\{\delta u_{1}^{1} \frac{\partial \phi_{1}}{\partial \xi}+\delta u_{1}^{2} \frac{\partial \phi_{2}}{\partial \xi}+\delta u_{1}^{3} \frac{\partial \phi_{3}}{\partial \xi}+\delta u_{1}^{4} \frac{\partial \phi_{4}}{\partial \xi}+\delta u_{1}^{5} \frac{\partial \phi_{5}}{\partial \xi}+\delta u_{1}^{6} \frac{\partial \phi_{6}}{\partial \xi}+\delta u_{1}^{7} \frac{\partial \phi_{7}}{\partial \xi}+\delta u_{1}^{8} \frac{\partial \phi_{8}}{\partial \xi}+\delta u_{1}^{9} \frac{\partial \phi_{9}}{\partial \xi}\right\} \\
\delta \varepsilon_{22}= & \frac{2}{y_{7}-y_{1}}\left\{\delta u_{2}^{\frac{1}{2}} \frac{\partial \phi_{1}}{\partial \eta}+\delta u_{2}^{2} \frac{\partial \phi_{2}}{\partial \eta}+\delta u_{2}^{3} \frac{\partial \phi_{3}}{\partial \eta}+\delta u_{2}^{4} \frac{\partial \phi_{4}}{\partial \eta}+\delta u_{2}^{5} \frac{\partial \phi_{5}}{\partial \eta}+\delta u_{2}^{6} \frac{\partial \phi_{6}}{\partial \eta}+\delta u_{2}^{7} \frac{\partial \phi_{7}}{\partial \eta}+\delta u_{2}^{8} \frac{\partial \phi_{8}}{\partial \eta}+\delta u_{2}^{9} \frac{\partial \phi_{9}}{\partial \eta}\right\} \\
\delta \varepsilon_{12}= & \frac{2}{y_{7}-y_{1}}\left\{\delta u_{1}^{1} \frac{\partial \phi_{1}}{\partial \eta}+\delta u_{1}^{2} \frac{\partial \phi_{2}}{\partial \eta}+\delta u_{1}^{3} \frac{\partial \phi_{3}}{\partial \eta}+\delta u_{1}^{4} \frac{\partial \phi_{4}}{\partial \eta}+\delta u_{1}^{5} \frac{\partial \phi_{5}}{\partial \eta}+\delta u_{1}^{6} \frac{\partial \phi_{6}}{\partial \eta}+\delta u_{1}^{7} \frac{\partial \phi_{7}}{\partial \eta}+\delta u_{1}^{8} \frac{\partial \phi_{8}}{\partial \eta}+\delta u_{1}^{9} \frac{\partial \phi_{9}}{\partial \eta}\right\}+ \\
& \frac{2}{x_{3}-x_{1}}\left\{\delta u_{2}^{1} \frac{\partial \phi_{1}}{\partial \xi}+\delta u_{2}^{2} \frac{\partial \phi_{2}}{\partial \xi}+\delta u_{2}^{3} \frac{\partial \phi_{3}}{\partial \xi}+\delta u_{2}^{4} \frac{\partial \phi_{4}}{\partial \xi}+\delta u_{2}^{5} \frac{\partial \phi_{5}}{\partial \xi}+\delta u_{2}^{6} \frac{\partial \phi_{6}}{\partial \xi}+\delta u_{2}^{7} \frac{\partial \phi_{7}}{\partial \xi}+\delta u_{2}^{8} \frac{\partial \phi_{8}}{\partial \xi}+\delta u_{2}^{9} \frac{\partial \phi_{9}}{\partial \xi}\right\}
\end{aligned}
$$

Starting with presumed initial plastic strain increment value, the boundary solution is found by using equation (2). Applying transformation method as given by Kane [3], the singularity terms in equation (1) is completely removed, hence the boundary solution can give an exact values. In order to avoid the second order singularity problem comes from strain increment formulation given in equation (3), the calculation of strain increment is proposed by using displacement increment equation given by equation (1). The singularity terms arises from the last integral in the equation (1) is completely removed by applying transformation method given by Kane [3]. Then, the displacement increment value at all internal nodes in the structure can be calculated by using equation (1) and the strain increment at all internal nodes can be found from equation (10). The calculation of strains by this technique only involve the first order or weakly singularity problem and does not involved the second order singularity problem. 


\section{Numerical implementation}

In order to verify the strain incremental formulation proposed in Section 4, a simple elastoplasticity analysis is conducted to timber beam structure shown in Figure 2. 64 boundary elements and 128 quadrangular cells with 585 internal nodes placed at suspected plastic region were applied. The elastoplasticity analysis was applied using boundary element method. The timber material is assumed behaves orthotropic property, the elastic parameters are given as follow: $E_{x}=16388 \mathrm{~N} / \mathrm{mm}^{2}, E_{y}=1600 \mathrm{~N} / \mathrm{mm}^{2}, \mathrm{G}_{\mathrm{xy}}=71 \mathrm{~N} / \mathrm{mm}^{2}, v=0.2$.

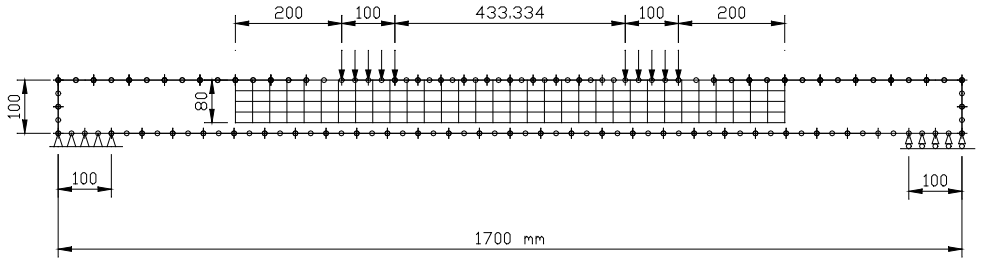

Figure 2. Boundary element and internal cell discretisation for timber beam structure

For the plastic yield criteria of the material, it is assumed that the yield locus is straight and the yield stresses are different values in $\mathrm{x}$ and $\mathrm{y}$ directions, see Figure 3. The flow rule is assumed obeys perfectly plastic property, where the plastic strain increment vector is outward normal to the yield locus.

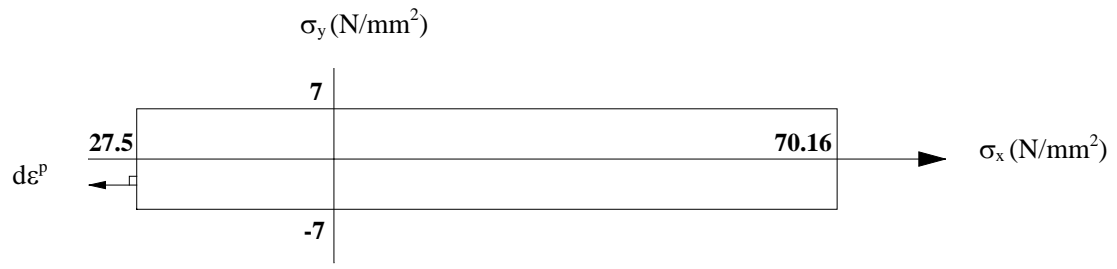

Figure 3. Presumed Yield locus diagram for timber 
The chosen number of elements at the boundary provides good accuracy of stresses and displacements at all internal nodes in the elastic stage. The chosen number of internal cells was provides satisfactory convergence of displacements and stresses at all internal nodes in the plastic stage.

Based on boundary element analysis results in the plastic stage, two typical stress distribution across the beam's depth of the beam are plotted and shown in Figure 4. The first stress distribution, namely as s.d.1, was predicted based on boundary element method using strain formulation given in Section 4. The second stress distribution, namely as s.d.2, was predicted by using boundary element method based on strain formulation proposed by Telles and Brebbia (refer Section 3). For the s.d. 1 curve, the stress areas at compression side and tension side are approximately equal and shows that the equilibrium of internal forces over the beam's cross section is satisfied. The neutral axis is also shifted down from the mid-depth position of the beam to maintain equilibrium of internal forces within the beam's cross section.

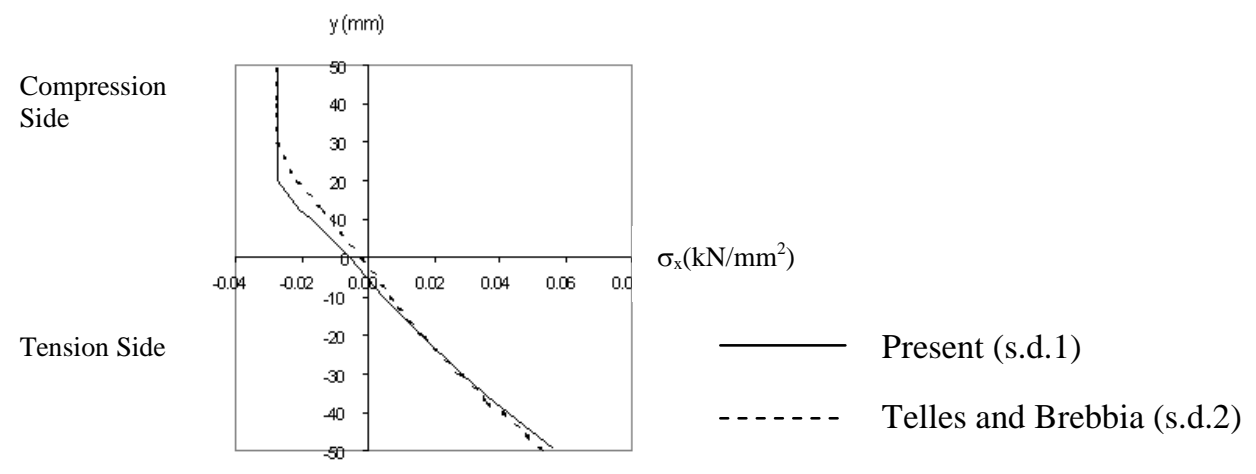

Figure 4. Stress distribution across the beam's depth at traction equal to $1.94 \times 10^{-3}$ $\mathrm{kN} / \mathrm{mm}^{2}$ (Plastic Stage)

Based on the result of boundary element analysis, the traction-deflection relationship is plotted and shown in Figure 5. The predicted curve using strain formulation given in Section 4 (present) is linear at elastic stage and indicated by the curve OA in Figure 5. After the yield limit of the material was reached at A, the slope of the curve is gradually changed and decreased and shown by the curve $\mathrm{AB}$ in Figure 5.

A well known properties of timber are elastic under tension stresses and elastoplastic under compression stresses [8]. The fully plastic zone at compression side (about half depth of the beam) (refer also Figure 4) is achieved when the traction value approach $0.00382 \mathrm{kN} / \mathrm{mm}^{2}$ and indicated by the point B in Figure 5. The linear stress distribution in the tension side (see Figure 4) indicate that the tension side of the beam is remained under fully elastic stage. Since the fully plastic stage at compression side and fully elastic stage at tension side of the beam were maintained in the model, the 
traction at point $\mathrm{B}$ in Figure 5 is a maximum value for the timber's beam and can not be exceeded beyond this point. As the timber property is partially plastic with the formation of wrinkle of timber's fibre at compression side of the beam during the plastic stage, the failure of the beam can occur at any time when the traction reach any point on the curve $\mathrm{AB}$ in Figure 5.

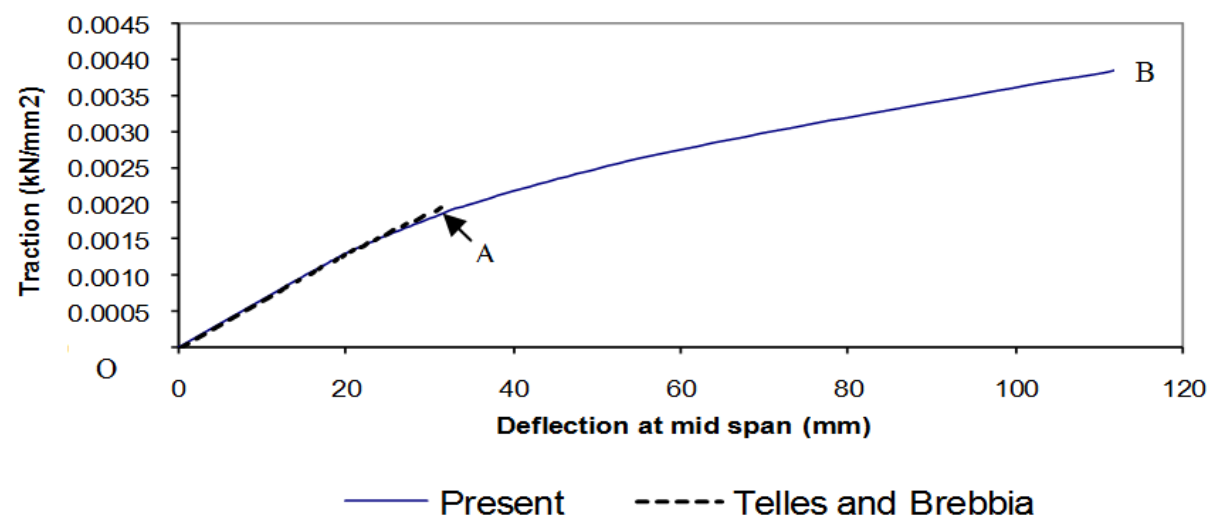

Figure 5. Traction-deflection curve

From Figure 5, the traction-deflection curve predicted using strain formulation proposed by Telles and Brebbia (equation 6 and 4) is terminated and ended at point A. For this case, the maximum traction at A is $1.94 \times 10^{-3} \mathrm{kN} / \mathrm{mm}^{2}$. The plastic analysis can not be proceeded because the convergence of plastic strain increment can not be satisfied.

The application of second order singularity for the strain increment calculation in the boundary element method is a main reason the convergence of plastic strain increment can not be satisfied. In the considered case for elastoplasticity plane stress beam, the application of second order singularity problem is proposed to be avoided.

\section{Conclusion}

To conclude this paper, the following remarks are noted:

1) The application of proposed formulation for the strain increment prediction in the boundary element method does not involved the application of second order singularity problem.

2) By using the proposed strain formulation, the convergence of plastic strain increment can be satisfied. The equilibrium of internal forces over the beam's cross section can be fulfilled and the continuity of traction-deflection curve from linear stage to plastic stage can be achieved.

3) The application of second order singularity problem in the elastoplasticity plane stress beam is proposed to be avoided. 


\section{References}

[1] Telles, J.C.F. and Brebbia, C.A., Elastic / Viscoplastic problems using boundary Elements, Int. J. Mech. Sci., 24, 605-618, 1982.

[2] Telles, J.C.F. and Brebbia, C.A., Boundary Elements: New Developments in Elastoplastic Analysis, Appl. Math. Modelling, 5, 376-382, 1981.

[3] Kane, J.H., Boundary Element Analysis in Engineering Continuum Mechanics, Prentice Hall, New Jersey, 1994.

[4] Telles, J.C.F., and Brebbia, C.A., On the Application of the Boundary Element Method to Plasticity, Appl. Math. Modelling, 3, 466-470, 1979.

[5] Guiggiani, M. and Gigante, A., A General Algorithm for Multidimensional Cauchy Principal Value Integrals in the Boundary Element Method, J. Applied Mechanics, 57, 906-915, 1990.

[6] Leitao, V., Aliabadi, M.H. and Rooke, D.P., The Dual Boundary Element Formulation for elastoplastic Fracture Mechanics, Int. J. Num. Meth. in Eng., 38, 315333, 1995.

[7] Swedlow, J.L. and Cruse, T.A., Formulation of Boundary Integral Equations for Three-Dimensional Elastoplastic Flow, International Journal of Solids and Structures, 7, 1673-1683, 1971.

[8] Malhotra, S.K. and Bazan, I.M.M., Ultimate Bending Strength Theory for Timber Beams, Wood Science, 12:7, 50-63, 1980. 